\title{
A cavity-QED scheme for Heisenberg-limited interferometry
}

\author{
DAVID VITALI ${ }^{\dagger}$, STEFAN KUHR $^{\ddagger}$, MICHEL BRUNE ${ }^{\ddagger}$, JEAN-MICHEL RAIMOND $^{\ddagger}$ \\ ${ }^{\dagger}$ Dipartimento di Fisica, Università di Camerino, \\ via Madonna delle Carceri, I-62032 Camerino (MC), Italy \\ ${ }^{\ddagger}$ Laboratoire Kastler Brossel, Département de Physique de l’Ecole Normale Supérieure, \\ 24 rue Lhomond, F-75231 Paris Cedex 05, France \\ (February 01, 2006)
}

\begin{abstract}
We propose a Ramsey interferometry experiment using an entangled state of $N$ atoms to reach the Heisenberg limit for the estimation of an atomic phase shift if the atom number parity is perfectly determined. In a more realistic situation, due to statistical fluctuations of the atom source and the finite detection efficiency, the parity is unknown. We then achieve about half the Heisenberg limit. The scheme involves an ensemble of circular Rydberg atoms which dispersively interact successively with two initially empty microwave cavities. The scheme does not require very high-Q cavities. An experimental realization with about ten entangled Rydberg atoms is achievable with state of art apparatuses.
\end{abstract}

\section{Introduction}

Quantum limits to noise in spectroscopy [1,2] and interferometry [3, 4, 5, 6, have attracted an increasing interest in the last years. The precision of an interferometric phase measurement is ultimately limited by the "Heisenberg uncertainty relation" between energy and time 17,8, 9, 10. However, this limit can only be reached when $N$ entangled particles are used. This represents a major experimental difficulty which has been overcome only very recently. In Ref. 8] three beryllium ions, and more recently in Ref. 9] six beryllium ions have been prepared in a maximally entangled state and used in a Ramsey spectroscopy experiment achieving a sensitivity close to the Heisenberg limit. In Refs. [11,12] experiments involving respectively three and four maximally entangled photons are reported, clearly showing interference fringes three and four times narrower than those obtained in an experiment with uncorrelated photons. Here, we propose an easy to implement cavity scheme, involving significantly more particles $(N>10)$.

The aim of a typical interferometry experiment is the measurement of the relative phase $\varphi$ between two quantum states $|g\rangle$ and $|e\rangle$ of a two level atom. The standard approach is the Ramsey interferometry technique [13, in which a first $\pi / 2$ pulse produces a superposition of $|g\rangle$ and $|e\rangle$. After a waiting time, the relative phase is probed with a second $\pi / 2$ pulse. The Ramsey setup is formally equivalent to an optical Mach-Zehnder interferometer, with the two $\pi / 2$ Ramsey pulses corresponding to the input and output $50-50$ beam splitters. The atomic phase shift corresponds to the differential phase shift between the two arms of the interferometer 2, 4. The uncertainty $\Delta \varphi$ of this phase measurement is limited by counting statistics to $\Delta \varphi=1 / \sqrt{N}$, where $N$ is the number of detected particles (atoms for Ramsey spectroscopy, photons in the Mach-Zehnder interferometer) [7].

This limit holds, however, only if the $N$ particles are uncorrelated, i. e. in a separable quantum state. If, instead, the $N$ particles are quantum correlated and share some entanglement (which is equivalent to say that they are spin-squeezed [5, 14]), quantum noise sets a fundamental limit to the phase uncertainty, $\Delta \varphi=1 / N$. This ultimate limit is called the Heisenberg limit and is achieved when a maximally entangled state of the $N$ particles is used and appropriately measured [7, 10].

We present here a variant of a Ramsey interferometry experiment which approaches the ultimate Heisenberg-limited resolution and which is realizable with present technology. It involves a collective interaction of $N$ Rydberg atoms with two high-Q microwave cavities. In the limit of a large atom-cavity detuning (dispersive interaction), the $N$ atoms are prepared in a maximally entangled state, i.e. in an 
atomic Schrödinger cat state after their collective interaction with the first cavity [15. This state is sensitive to a Stark phase shift $\varphi$ applied between the two cavities. The interaction with the second cavity reads out this phase shift. The final measurement of the atomic population reveals interference fringes $N$ times narrower than those achievable using non-entangled atoms.

The outline of the paper is as follows. In Sec. 2 the scheme of the experiment is described in detail. In Sec. 3 we calculate the interference signal resulting from averaging over the fluctuations of the atom number. In Sec. 4 we present a modified scheme, which improves this averaged signal. Finally, in Sec. 5 a conditional detection scheme further improving the sensitivity of the detection of the phase shift is illustrated, while Sec. 6] contains concluding remarks.

\section{Scheme of the interferometry experiment}

A schematic description of the proposed interferometry experiment is shown in Fig. 1, It involves a collection of $N$ two-level atoms simultaneously crossing a set of microwave cavities. The two relevant atomic levels are circular Rubidium Rydberg states $|e\rangle$ and $|g\rangle$ with principal quantum numbers 51 and 50, and a transition frequency $\omega_{e g} / 2 \pi=51 \mathrm{GHz}$. The circular Rydberg states are characterized by a very long lifetime $(\sim 30 \mathrm{~ms})$ and a very large dipole moment [16]. The atoms are simultaneously prepared in zone $B$ by the excitation of a velocity-selected atomic beam effusing from an oven. The $N$ atoms sample, whose size is negligible at the scale of the millimeter wavelength $\lambda=2 \pi c / \omega_{e g}$, crosses the arrangement of microwave cavities at the same velocity $v$. Three "Ramsey zones", $R_{j}(j=1,2,3)$, are separated by two identical microwave Fabry-Perot cavities $C_{1}$ and $C_{2}$, which have a high quality factor $Q$. The three Ramsey zones are microwave cavities with low $Q$, in which the Rydberg atoms can be subjected to classical resonant pulses, generated by standard phase-locked microwave sources. The three cavities $R_{j}$ are resonant with the $g \leftrightarrow e$ transition, while the high-Q cavities $C_{1}$ and $C_{2}$ are off-resonant. The atom-cavity detunings are $\delta_{i}=\omega_{e g}-\omega_{\mathrm{c}, i}\left(\omega_{\mathrm{c}, i}\right.$ is the mode angular frequency of cavity $\left.i\right)$. The atoms are finally detected in $D$ by a state selective field-ionization detector, able to count both the number of atoms in $|e\rangle, N_{e}$ and in $|g\rangle, N_{g}$.

The $N$ atoms collectively interact with the quantized radiation mode of $C_{1}$ and $C_{2}$, where they are symmetrically coupled [17. The interaction of the atomic system with cavity $i$, in a frame rotating at $\omega_{e g}$, is described by the Tavis-Cummings Hamiltonian [18.

$$
\hat{H}_{I, i}(t)=-\hbar \delta_{i} \hat{a}_{i}^{\dagger} \hat{a}_{i}+i \hbar \frac{\Omega_{i}(t)}{2}\left(\hat{a}_{i}^{\dagger} \hat{J}_{-}-\hat{a}_{i} \hat{J}_{+}\right)
$$

where we have introduced the angular momentum Dicke operators $\hat{J}_{-}=\sum_{i}^{N}|g\rangle_{i}\langle e|, \hat{J}_{+}=\left(\hat{J}_{-}\right)^{\dagger}$ and

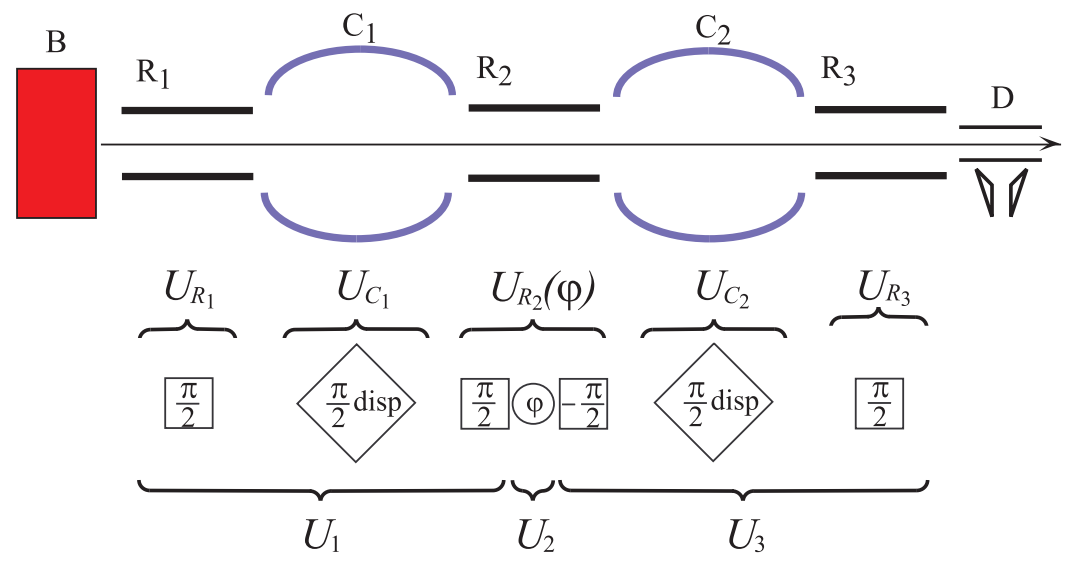

Figure 1. Schematic description of the proposed cavity-QED interferometry experiment. The $N$ atoms are prepared in the circular Rydberg state $|g\rangle$ in $B$, then cross the three classical field zones $R_{j}$ and the two high-Q cavities $C_{j}$, and are finally detected in $D$. The squares denote $\pi / 2$ Rabi pulses in $R_{j}$, the circle denotes the tunable Stark shift pulse in $R_{2}$, and the diamonds denote the dispersive atom-cavity interaction in $C_{j}$. 
$\hat{J}_{z}=(1 / 2) \sum_{i}|e\rangle_{i}\langle e|-| g\rangle_{i}\langle g|$ [17]. In Eq. (11), $\hat{a}_{i}$ is the annihilation operator of the quasi-resonant mode in $C_{i}$, and $\Omega_{i}(t)=\Omega_{0} \exp \left[-v^{2} t^{2} / w^{2}\right]$ is the vacuum Rabi frequency describing the atom-field interaction. The time dependency is due to the Gaussian profile of the radiation mode with waist $w$ by the moving atomic sample.

We are interested in the dispersive limit in which the two cavities $C_{1}$ and $C_{2}$ are detuned far from the atomic transition, i.e., $\delta \gg \Omega_{0} \sqrt{N}, \gamma_{\mathrm{c}}\left(\gamma_{\mathrm{c}}\right.$ is the cavity decay rate). Using cryogenic techniques together with appropriate "cooling" atoms [16, one can approach the zero temperature limit and therefore we shall assume a mean thermal photon number of zero from now on. Neglecting the trivial constant of motion $\hat{J}^{2}=(N / 2)(N / 2+1)$, Eq. (11) is approximated as

$$
\hat{H}_{\mathrm{disp}, i}(t)=-\hbar \frac{\Omega_{i}(t)^{2}}{4 \delta_{i}}\left(\hat{J}_{z}^{2}-\hat{J}_{z}\right),
$$

explicitly showing that, in the dispersive limit, the atomic excitation $\hat{J}_{z}$ and the photon excitation $\hat{a}_{i}^{\dagger} a_{i}$, independently, are constants of motion. The term $\hat{J}_{z}^{2}$ in Eq. (2) is responsible for the generation of atomic entanglement and spin squeezing [5]. This is reminiscent of the optical Kerr effect, which is characterized by a quadratic term in the photon number. In the atomic as well as in the optical case, this quadratic term is able, for particular values of the interaction time, to generate "Schrödinger cats", i.e., quantum superpositions of coherent states with different classical phases [15, 19, 20.

We adopt the compact representation of an atomic coherent state with orientation on a Bloch sphere $(\theta, \phi),(0 \leq \theta \leq \pi, 0 \leq \phi<2 \pi)$, introduced in [21],

$$
\begin{aligned}
|\theta, \phi\rangle & =e^{-i \theta\left(\hat{J}_{x} \sin \phi-\hat{J}_{y} \cos \phi\right)}|J,-J\rangle \\
& =\sum_{m=-J}^{J}\left(\begin{array}{c}
2 J \\
J+m
\end{array}\right)\left(\sin \frac{\theta}{2}\right)^{J+m}\left(\cos \frac{\theta}{2}\right)^{J-m} e^{-i \phi(J+m)}|J, m\rangle,
\end{aligned}
$$

where the so-called Dicke states $|J, m\rangle$ are the eigenstates of $\hat{J}_{z}$, and $\hat{J}^{2}$ with respective eigenvalues $m$ and $J(J+1)$.

The atoms are initially prepared in the atomic coherent state $|\psi\rangle_{0}=\prod_{i}|g\rangle_{i}=|0,0\rangle$ and are then subjected to a sequence of unitary operations $U_{R_{i}}(i=1,2,3)$ and $U_{C_{j}}(j=1,2)$ to generate the final state $|\psi\rangle_{\text {final }}$ (see Figs. 1] and 2):

$$
|\psi\rangle_{\text {final }}=U_{R_{3}} U_{C_{2}} U_{R_{2}}(\varphi) U_{C_{1}} U_{R_{1}}|\psi\rangle_{0}
$$

As a first step, the atoms undergo in $R_{1}$ a $\pi / 2$ pulse, described by the unitary operator $U_{R_{1}}=$ $\exp \left\{i \pi \hat{J}_{y} / 2\right\}$. It aligns the collective Bloch vector along the $x$-axis, generating the atomic coherent state

$$
|\psi\rangle_{R_{1}}=U_{R_{1}}|\psi\rangle_{0}=|\pi / 2,0\rangle
$$

The interaction with classical microwave pulses only changes the orientation of an atomic coherent state on the Bloch sphere 21] but the $N$ atoms remain disentangled. The atomic entanglement is produced in $C_{1}$ by dispersive interaction with the radiation mode, which is initially prepared in the vacuum state. As shown in [15], for specific values of the interaction time, an initial atomic coherent state $|\theta, \phi\rangle$ is transformed into a superposition of a finite number of coherent states with the same $\theta$ and equally spaced $\phi$ values. An atomic Schrödinger cat state with two components is the maximally entangled $N$-partite GHZ state corresponding to the superposition of two atomic coherent states with opposite orientations on the Bloch sphere, $\left[|\theta, \phi\rangle+e^{i \beta}|\pi-\theta, \phi+\pi\rangle\right] / \sqrt{2}$. In our proposal we obtain such an atomic cat state when [15]

$$
\int d t \frac{\Omega_{1}(t)^{2}}{4 \delta_{1}}=\frac{\pi}{2}
$$




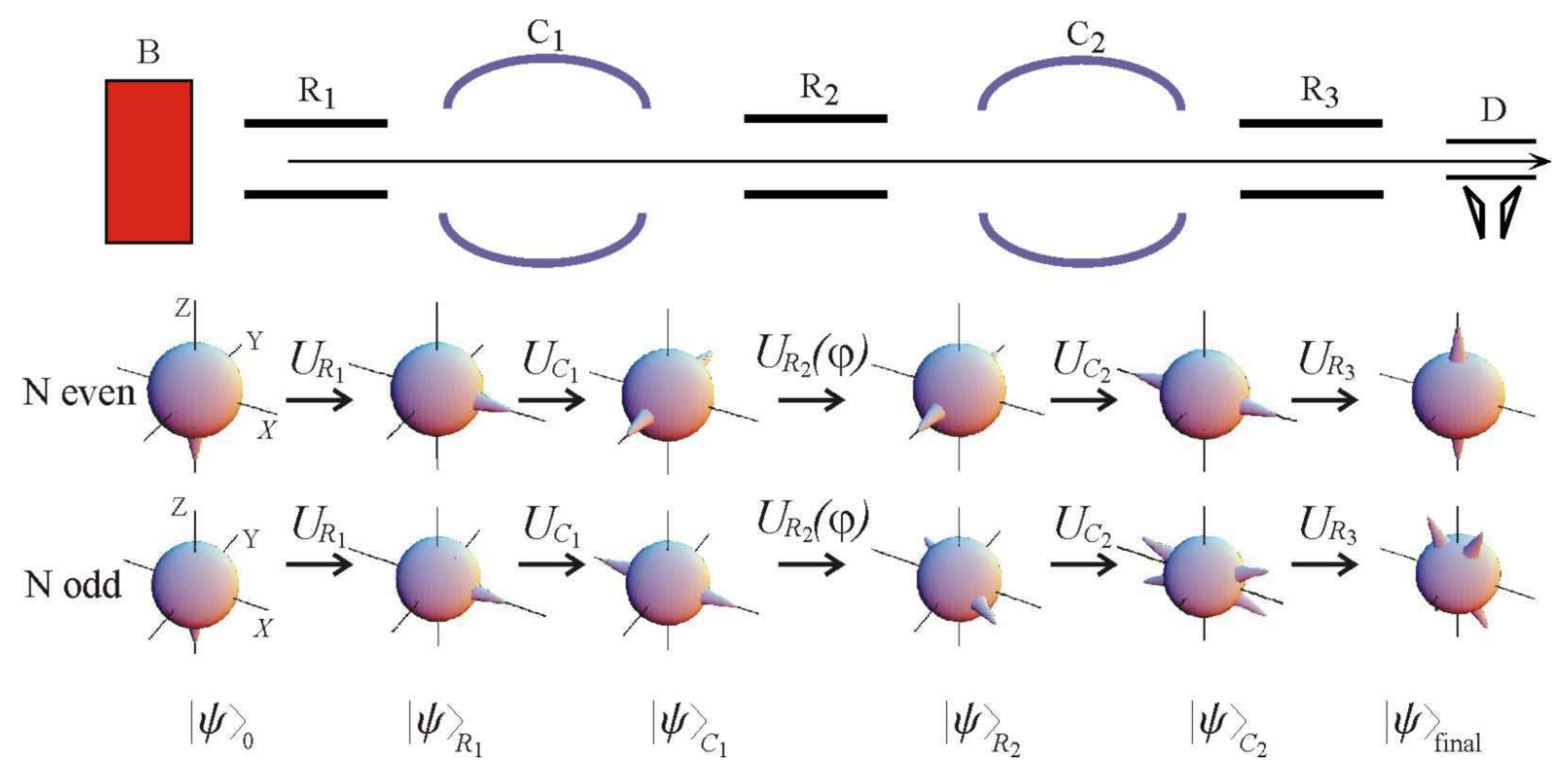

Figure 2. Schematic description of the state of the $N$ Rydberg atoms on a Bloch sphere at the various stages of the interferometry experiment, both for even $N$ and for odd $N$. For both parities and at every stage, the $N$ atoms are in a superposition of atomic coherent states (see Eqs. 81- 13 and Eqs. 17- 18 for the explicit expression of the atomic states in the two cases).

When this condition is satisfied, the interaction in $C_{1}$ is represented by the unitary operator

$$
U_{C_{1}}=\exp \left[i \frac{\pi}{2}\left(\hat{J}_{z}^{2}-\hat{J}_{z}\right)\right]
$$

and the state $|\psi\rangle_{C_{1}}=U_{C_{1}}|\psi\rangle_{R_{1}}$ at the exit of $C_{1}$ becomes [15]

$$
|\psi\rangle_{C_{1}}^{\text {even }}=\frac{1}{\sqrt{2}}\left[e^{i \frac{\pi}{4}}\left|\frac{\pi}{2}, \frac{\pi}{2}\right\rangle+(-1)^{\frac{N}{2}} e^{-i \frac{\pi}{4}}\left|\frac{\pi}{2}, \frac{3 \pi}{2}\right\rangle\right]
$$

if $N$ is even, and

$$
|\psi\rangle_{C_{1}}^{\text {odd }}=\frac{1}{\sqrt{2}}\left[e^{i \frac{\pi}{4}}\left|\frac{\pi}{2}, \pi\right\rangle+(-1)^{\frac{N-1}{2}} e^{-i \frac{\pi}{4}}\left|\frac{\pi}{2}, 0\right\rangle\right]
$$

if $N$ is odd. This means that we have a cat state directed along the $y$-axis for even $N$ and along the $x$-axis for odd $N$ (see Fig. 2).

The goal of the interferometry experiment is to detect a variable phase difference $\varphi$ between the two components of the cat state which is applied in $R_{2}$ by a Stark pulse. This pulse induce a dephasing between the states $|g\rangle$ and $|e\rangle$, aligned along the $z$-axis. Thus, we have to rotate the cat state, aligned along the $y$-axis, to the $z$-axis prior to the application of the Stark pulse and then rotate it back into its initial direction. For the case of even $N$, this corresponds to the pulse sequence

$$
U_{R_{2}}(\varphi)=e^{i \pi \hat{J}_{x} / 2} e^{i \varphi \hat{J}_{z}} e^{-i \pi \hat{J}_{x} / 2}
$$

which is equivalent to $U_{R_{2}}(\varphi)=\exp \left\{i \varphi \hat{J}_{y}\right\}$. We obtain

$$
|\psi\rangle_{R_{2}}^{\text {even }}=U_{R_{2}}(\varphi)|\psi\rangle_{C_{1}}^{\text {even }}=\frac{1}{\sqrt{2}}\left[e^{i \frac{\pi}{4}+i \frac{N \varphi}{2}}\left|\frac{\pi}{2}, \frac{\pi}{2}\right\rangle+(-1)^{\frac{N}{2}} e^{-i \frac{\pi}{4}-i \frac{N \varphi}{2}}\left|\frac{\pi}{2}, \frac{3 \pi}{2}\right\rangle\right]
$$

Thus, the two state components have acquired opposite phase shifts $\pm N \varphi / 2$, proportional to $N$. 
Then the atoms enter $C_{2}$, where they undergo the same transformation as in $C_{1}, U_{C_{2}}=U_{C_{1}}$. It is easy to see that the atomic state becomes:

$$
|\psi\rangle_{C_{2}}^{\text {even }}=-\sin \left(\frac{N \varphi}{2}\right)\left|\frac{\pi}{2}, \pi\right\rangle+(-1)^{\frac{N}{2}} \cos \left(\frac{N \varphi}{2}\right)\left|\frac{\pi}{2}, 0\right\rangle .
$$

It is still an entangled atomic Schrödinger cat state, directed along the $x$-axis, with $\varphi$-dependent amplitudes. Since we always detect atomic energies, i.e., the number of atoms in $|g\rangle$ or $|e\rangle$, we use a final $\pi / 2$ pulse in zone $R_{3}$ before the detection, $U_{R_{3}}=U_{R_{1}}$. The cat state of Eq. (12) is rotated around the $y$-axis and put along the $z$ direction, so that one obtains the final state

$$
|\psi\rangle_{\text {final }}^{\text {even }}=-\sin \left(\frac{N \varphi}{2}\right)|\theta=0\rangle+(-1)^{\frac{N}{2}} \cos \left(\frac{N \varphi}{2}\right)|\theta=\pi\rangle
$$

A measurement of the expectation value of $\left\langle\hat{J}_{z}\right\rangle$ is done by counting the number of atoms in $|e\rangle \hat{=}|\theta=\pi\rangle$ and $|g\rangle \hat{=}|\theta=0\rangle$. Using the corresponding operators $\hat{N}_{e}=N / 2+\hat{J}_{z}$ and $\hat{N}_{g}=N / 2-\hat{J}_{z}$, we obtain

$$
\hat{J}_{z}=\frac{\hat{N}_{e}-\hat{N}_{g}}{2}
$$

The expectation value of $\hat{J}_{z}$ in state $|\psi\rangle_{\text {final }}^{\text {even }}$ is

$$
\left\langle\hat{J}_{z}\right\rangle^{\text {even }}=\frac{N}{2} \cos (N \varphi)
$$

This signal displays Ramsey fringes with unit contrast oscillating $N$ times faster than those corresponding to a single atom (see Fig. [3). To calculate the sensitivity of $\left\langle\hat{J}_{z}\right\rangle$ to changes of $\varphi$, we evaluate [22]

$$
\Delta \varphi=\frac{\Delta \hat{J}_{z}}{\left|\partial\left\langle\hat{J}_{z}\right\rangle / \partial \varphi\right|},
$$

where $\Delta \hat{J}_{z}=\sqrt{\left\langle\hat{J}_{z}^{2}\right\rangle-\left\langle\hat{J}_{z}\right\rangle^{2}}$ measures the fluctuations of the operator $\hat{J}_{z}$. Eq. (13) yields $\left\langle\hat{J}_{z}^{2}\right\rangle^{\text {even }}=N^{2} / 4$ so that, using Eqs. (15)-(16), one obtains $\Delta \varphi=1 / N$, independent of $\varphi$. This means that, for an even number of atoms, we obtain a $\sqrt{N}$ increase of sensitivity with respect to a standard Ramsey experiment involving $N$ uncorrelated atoms [7] and thus achieve the Heisenberg limit.

Grouping in a different way the unitary operations, we stress the link between this scheme and a genuine Ramsey interferometry experiment (see Fig. 1). We divide the sequence of operations into three parts $U_{i},(i=1,2,3)$. The first, $U_{1}=\exp \left\{-i \pi \hat{J}_{x} / 2\right\} U_{C_{1}} U_{R_{1}}$, consists of the $\pi / 2$ pulse in $R_{1}$, the dispersive interaction in $C_{1}$ and the first $\pi / 2$ pulse in $R_{2}$, (see curly brackets in Fig. (1). These three operations prepare the $N$ atoms, initially in the atomic coherent state $|\theta=0\rangle$, in the maximally entangled superposition $\left[e^{i \frac{\pi}{4}}|\theta=\pi\rangle+(-1)^{\frac{N}{2}} e^{-i \frac{\pi}{4}}|\theta=0\rangle\right] / \sqrt{2}$ (in the case of $N$ even). The second part corresponds to the application of the tunable phase $\varphi$ through the Stark shift pulse, $U_{2}=\exp \left\{i \varphi \hat{J}_{z}\right\}$. Finally, the third part, $U_{3}=U_{R_{3}} U_{C_{2}} \exp \left\{i \pi \hat{J}_{x} / 2\right\}$, consists of the second $\pi / 2$ pulse in $R_{2}$, the dispersive interaction in $C_{2}$ and the final $\pi / 2$ pulse in $R_{3}$. The operations $U_{1}$ and $U_{3}$ are thus "Super-Ramsey pulses", corresponding to the $\pi / 2$ pulses of a classical Ramsey experiment.

In the preceding discussion we only considered the case of an even atom number. For odd $N$ values, the state $|\psi\rangle_{R_{2}}$ at the exit of $R_{2}$ becomes

$$
|\psi\rangle_{R_{2}}^{\mathrm{odd}}=\frac{1}{\sqrt{2}}\left[e^{i \frac{\pi}{4}}\left|\frac{\pi}{2}-\varphi, \pi\right\rangle+(-1)^{\frac{N-1}{2}} e^{-i \frac{\pi}{4}}\left|\frac{\pi}{2}+\varphi, 0\right\rangle\right]
$$

for $|\varphi| \leq \pi / 2$. For larger values of $|\varphi|$, similar expressions can be derived. $|\psi\rangle_{R_{2}}^{\text {odd }}$ is still a maximally 


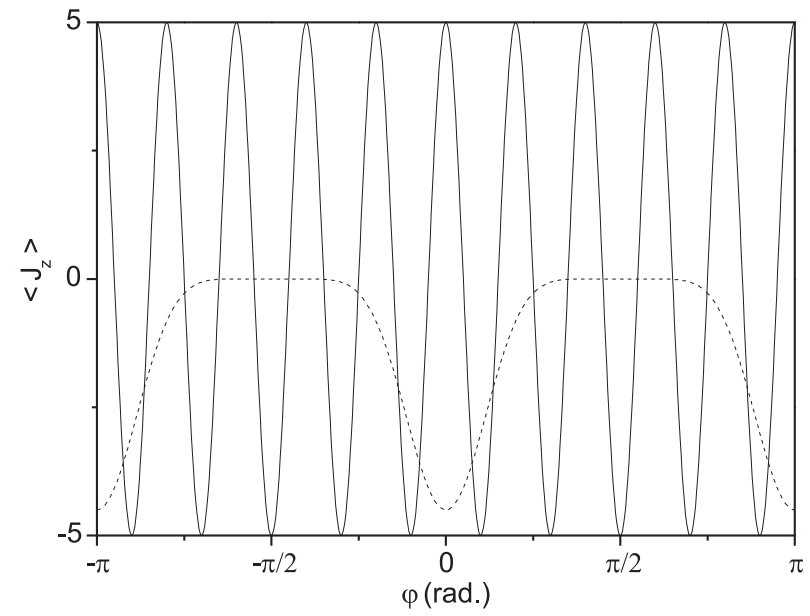

Figure 3. Interferometric signal $\left\langle\hat{J}_{z}\right\rangle$ after the entire Ramsey pulse sequence, according to Eqs. (15) and (19). Shown are the cases for even $(N=10$, solid line) and odd $(N=9$, dashed line) atom number. The signals differ significantly in shape depending on the parity of the atom number.

entangled cat state, however oriented in the $x z$ plane at an angle $\varphi$ with the $x$ axis. The Stark shift pulse does not apply a relative phase on the state components, but rather rotates them around the $z$ axis by an angle $\varphi$. As a consequence, the final state of the $N$ atoms after $R_{3}$ is very different from that of Eq. (13):

$$
|\psi\rangle_{\text {final }}^{\text {odd }}=\frac{1}{2}\left[|\varphi, \pi\rangle+|\varphi, 0\rangle-i(-1)^{\frac{N-1}{2}}(|\pi-\varphi, \pi\rangle-|\pi-\varphi, 0\rangle)\right]
$$

It is a superposition of four coherent states, all in the $x z$ plane (see Fig. 2). Using the properties of atomic coherent states, it is possible to see that this state yields an interferometric signal given by

$$
\left\langle\hat{J}_{z}\right\rangle^{\text {odd }}=-\frac{N}{2}(\cos \varphi)^{N-1}
$$

very different from that for even $N$ (see Fig. 3). It does not display any fast oscillation, but only dips at $\varphi=0, \pm \pi$, having a width inversely proportional to $\sqrt{N}$. Moreover Eq. (18) yields $\left\langle\hat{J}_{z}^{2}\right\rangle^{\text {odd }}=$ $\left(N^{2} \cos ^{2} \varphi+N \sin ^{2} \varphi\right) / 4$ so that one obtains the following phase sensitivity in the case of odd $N$

$$
\Delta \varphi^{\text {odd }}=\frac{\sqrt{N^{2}\left[1-(\cos \varphi)^{2 N-2}\right]-N(N-1) \sin ^{2} \varphi}}{N(N-1)|\sin \varphi||\cos \varphi|^{N-2}} .
$$

This expression is minimum at $\varphi=0, \pm \pi$, where $\Delta \varphi^{\text {odd }}=1 / \sqrt{N}$, implying that, for an odd number of atoms, the phase sensitivity is always worse, or at best equal, to that of a standard Ramsey experiment involving $N$ uncorrelated atoms [7].

\section{Interference signal averaged over the fluctuations of the atom number}

An experimental implementation of the scheme described above will be affected by the Poissonian fluctuations of the number of atoms and a non-ideal detection efficiency of the detectors, which makes it impossible to infer the parity of $N$. If we assume that we prepare $N$ Rydberg atoms with a Poissonian probability distribution with mean number $\bar{N}$ and average all detection events, the resulting interferometric signal is given by the average over the distribution of a signal given by Eq. (15) for even $N$, and by 
(a)

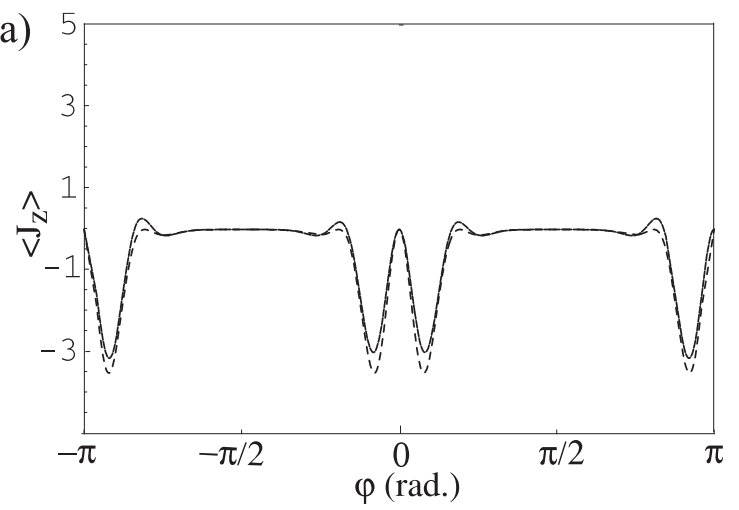

(b)

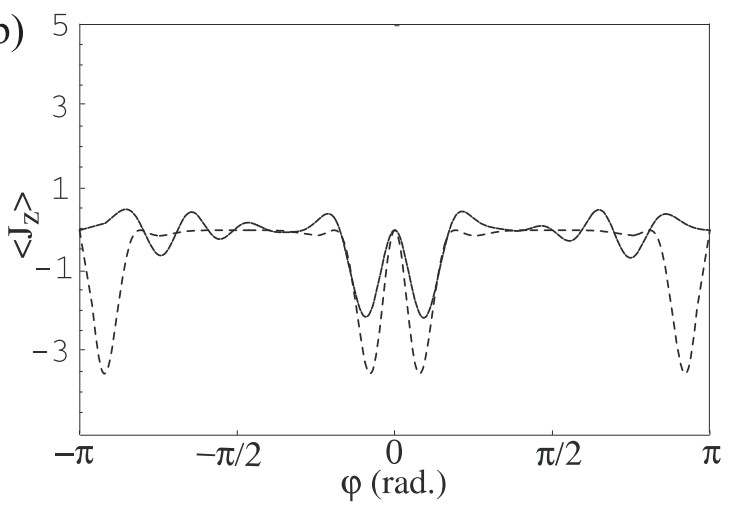

Figure 4. Interferometric signal $\left\langle\hat{J}_{z}\right\rangle$ for Poisson distributed number of atoms, with mean number $\bar{N}=10$. The dashed lines show Eq. 21, valid in the dispersive limit. The full line is the numerical solution of the exact dynamics out of the dispersive regime. (a) $\delta / \Omega_{0}=9.27, v=40 \mathrm{~m} / \mathrm{s}$. (b) $\delta / \Omega_{0}=2.06, v=180 \mathrm{~m} / \mathrm{s}$ (see text for the other parameters).

Eq. (19) for odd $N$. The corresponding expression is evaluated analytically:

$$
\left\langle\hat{J}_{z}\right\rangle=\frac{\bar{N}}{2} e^{-\bar{N}}\left\{\sinh \left(u_{\varphi}\right) \cos \left(v_{\varphi}\right) \cos \varphi-\cosh \left(u_{\varphi}\right)\left[1+\sin \left(v_{\varphi}\right) \sin \varphi\right]\right\}
$$

with $u_{\varphi}=\bar{N} \cos \varphi, v_{\varphi}=\bar{N} \sin \varphi$. The behavior of this signal for $\bar{N}=10$ is shown in Fig. 4(a) (dashed line), where it is compared with the prediction of a numerical solution of the dynamics driven by the exact Hamiltonian [Eq. (1)] in the presence of cavity damping (full line). The fast oscillating fringes of Eq. (15) except the central one at $\varphi=0$ are degraded by the incoherent average over different frequencies. In addition, the contribution of the experimental runs with an odd atom number reduces the height of the central fringe.

The agreement of Eq. (21) with the numerical simulation is good since we have chosen a parameter region corresponding to the dispersive regime of large detunings. Using the values of Ref. 16, i.e., $\Omega_{0}=$ $0.31 \times 10^{6} \mathrm{~s}^{-1}, w=6 \mathrm{~mm}$, an atomic decay rate $\gamma_{\mathrm{a}}=33.3 \mathrm{~s}^{-1}$ and a cavity decay rate $\gamma_{\mathrm{c}}=10^{3} \mathrm{~s}^{-1}$, Fig. 4(a) refers to $\delta / \Omega_{0}=9.27$, i.e., $\delta=2.87 \times 10^{6} \mathrm{~s}^{-1}$, which, taking the condition of Eq. (6) into account, implies an atomic velocity $v=40 \mathrm{~m} / \mathrm{s}$. The resulting signal $\left\langle\hat{J}_{z}\right\rangle$ is periodic with period $\pi$. Its contrast is smaller than $1 / 2$ because of the signal suppression in the wrong parity case. Despite that, this signal still scales with the number of atoms as the Heisenberg limit, thanks to the peak at $\varphi=0$, whose width is inversely proportional to $\bar{N}$, as it can be seen from the approximate expression of Eq. (21) at small $\varphi$

$$
\left\langle\hat{J}_{z}\right\rangle \simeq-\frac{\bar{N}}{2} \exp \left(-\frac{\bar{N} \varphi^{2}}{2}\right) \sin ^{2}\left[\frac{\varphi(\bar{N}+1)}{2}\right] .
$$

The exact phase sensitivity is provided by Eq. (16), whose explicit expression in the case of Poissondistributed number of atoms can be obtained from Eq. (21) and the Poisson average of the squared momentum

$$
\left\langle\hat{J}_{z}^{2}\right\rangle=\frac{\bar{N}}{4}+\frac{\bar{N}^{2}}{8}\left(1+\cos ^{2} \varphi+e^{-2 \bar{N}} \sin ^{2} \varphi\right)
$$

The resulting expression is a cumbersome function of $\varphi$, achieving its minimum at $|\varphi| \simeq \pi /(2 \bar{N}+2)$, which also approximately corresponds to the points where the absolute value of the slope of the interferometric signal is maximum. At these phase shift values, $\Delta \varphi \simeq 2 / \bar{N}$, showing that at small values of $\varphi$ and in the dispersive regime, our scheme reaches $1 / 2$ of the Heisenberg limit despite the presence of a fluctuating number of atoms.

The low atomic velocity is hardly compatible with the state of the art experiments [16]. We thus consider the case of a more realistic velocity $v=180 \mathrm{~m} / \mathrm{s}$, for which we expect to have a reasonable flux of atoms. In this case the $\pi / 2$-pulse condition of Eq. (6) imposes a smaller detuning $\delta / \Omega_{0}=2.06$. The behavior of 
$\left\langle\hat{J}_{z}\right\rangle$ in this non-dispersive regime is shown in Fig. 4(b) (full line), where it is compared with the dispersive limit expression of Eq. (21) for $\bar{N}=10$ (dashed line). The fringes at $\varphi= \pm \pi$ have been washed out, while the central fringe at $\varphi=0$ is still visible, even though its contrast is now significantly reduced. The corresponding resolution in the estimation of $\varphi$ is now decreased and one has $\Delta \varphi \simeq 3.3 / \bar{N}$.

\section{Modified scheme with inversion of time evolution}

As shown in Fig. 4(b), the contrast of the interference fringes degrades as soon as we leave the dispersive limit of slow velocities and we consider the fluctuations of the number of atoms. However, it is possible to significantly improve the scheme if we avoid the suppression of the signal at $\varphi=0$ in the odd $N$ case (see Eq. (19)) which is responsible for the fact that the signal is practically never larger than zero. This can be achieved if the time evolution in $C_{2}$ is "inverted", i.e., $\hat{H}_{I, 2}(t) \rightarrow-\hat{H}_{I, 2}(t)$. In the dispersive limit, this inversion can be achieved by changing only the sign of the detuning $\delta_{2}$ through an appropriate Stark shift of the atomic levels. In such a case at $\varphi=0$ nothing happens in $R_{2}$, and the two interactions in $C_{1}$ and $C_{2}$ cancel each other. The atoms only undergo the two $\pi / 2$ pulses in $R_{1}$ and $R_{3}$ which rotate the state from $|\theta=0\rangle$ to $|\theta=\pi\rangle$. The final result is $\left\langle\hat{J}_{z}\right\rangle=N / 2\left(N_{e}=N, N_{g}=0\right)$ at $\varphi=0$ either in the even and in the odd case. Within the dispersive limit, we derive the state of the $N$ atoms in the presence of this time evolution inversion. Nothing changes for even $N$, i.e., Eqs. (12)-(15) remain valid, while in the odd $N$ case the final state is now given by

$$
|\psi\rangle_{\text {final }}^{\text {odd,inv }}=\frac{1}{2}\left[|\varphi, 0\rangle-|\varphi, \pi\rangle-i(-1)^{\frac{N-1}{2}}(|\pi-\varphi, \pi\rangle+|\pi-\varphi, 0\rangle)\right]
$$

yielding an interferometric signal

$$
\left\langle\hat{J}_{z}\right\rangle^{\text {odd,inv }}=\frac{N}{2}(\cos \varphi)^{N-1}
$$

Using Eq. (25), the resulting Poisson-averaged interferometric signal can be obtained by changing only the sign of the term $\cosh \left(u_{\varphi}\right)$ in the expression of Eq. (21), so that

$$
\left\langle\hat{J}_{z}\right\rangle^{\text {inv }}=\frac{\bar{N}}{2} e^{-\bar{N}}\left[\sinh \left(u_{\varphi}\right) \cos \left(v_{\varphi}\right) \cos \varphi+\cosh \left(u_{\varphi}\right)\left[1-\sin \left(v_{\varphi}\right) \sin \varphi\right]\right]
$$

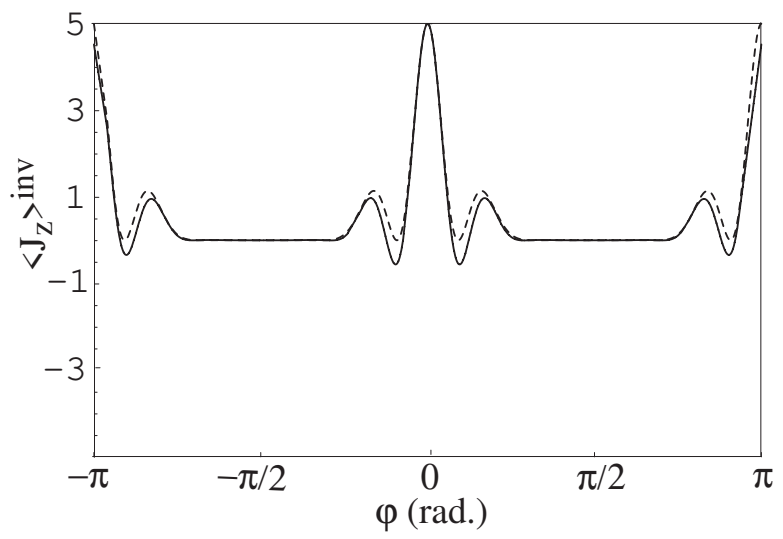

Figure 5. Interferometric signal $\left\langle\hat{J}_{z}\right\rangle^{\text {inv }}$ in the presence of the "inversion" of time evolution in $C_{2}$, for Poisson-distributed number of atoms with mean number $\bar{N}=10$. The dashed line refers to Eq. 26], which has been derived assuming the dispersive Hamiltonian of

Eq. (2), while the full line refers to the numerical solution of the exact dynamics in the case of large detuning, $\delta / \Omega_{0}=9.27$ and $v=40 \mathrm{~m} / \mathrm{s}$ (see text for the other parameters). 


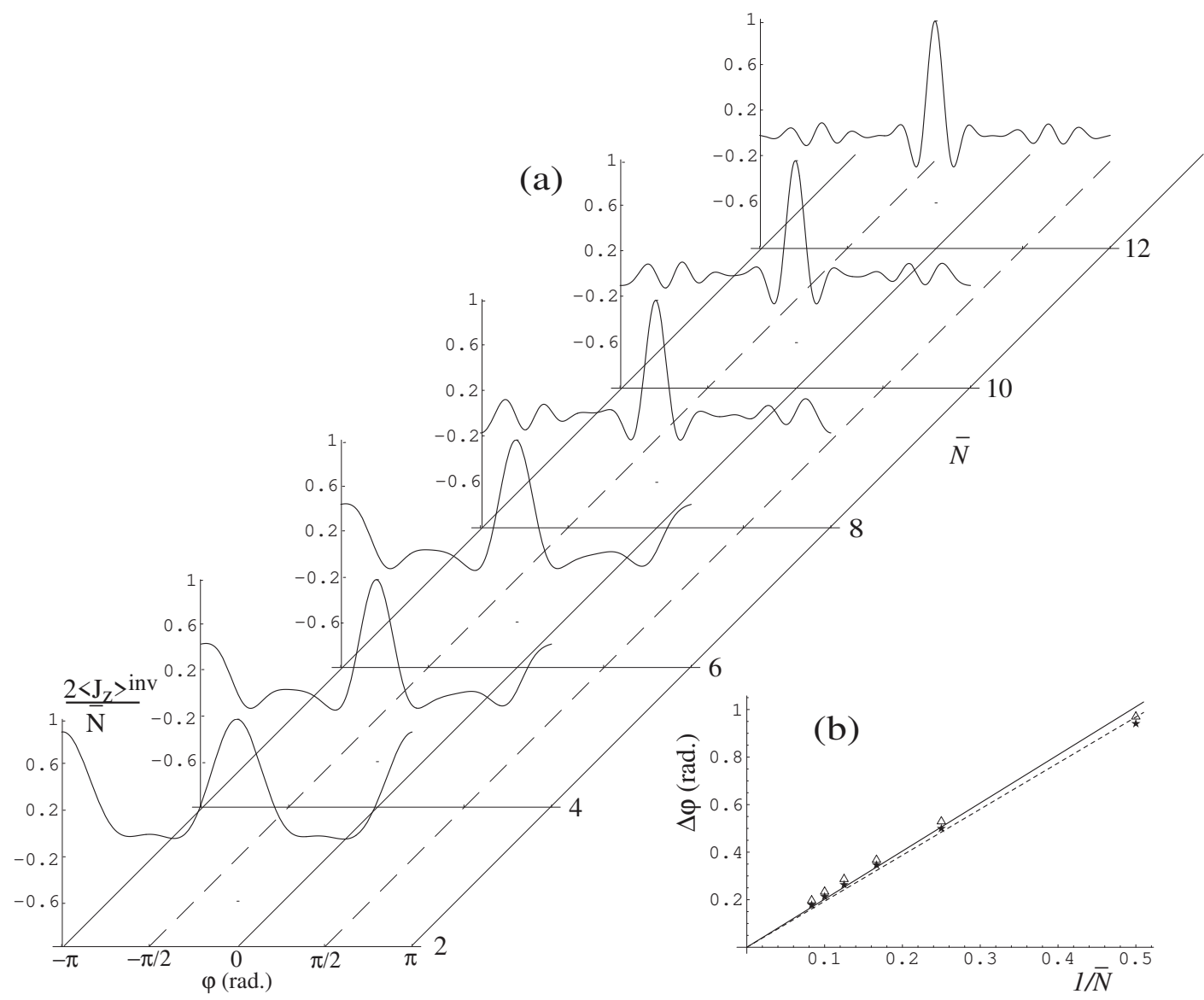

Figure 6. (a) Normalized interferometric signal $\left\langle 2 \hat{J}_{z}\right\rangle^{\text {inv }} / \bar{N}$ in the presence of the inversion in $C_{2}$, for Poisson-distributed number of atoms, with mean number $\bar{N}=2,4,6,8,10,12$. The curves show the numerical solution of the exact dynamics in the non-dispersive regime, with $\delta / \Omega_{0}=2.06$ and $v=180 \mathrm{~m} / \mathrm{s}$. (b) Phase uncertainty $\Delta \varphi$ as defined in Eq. (16) for the same parameter values, versus $1 / \bar{N}$. Stars refer to the minimum phase uncertainty and the dotted line is the least-squares linear fit of the data, while triangles refer to the phase uncertainty at the point of maximum absolute slope of the signal and the full line is the least-squares linear fit of the corresponding data.

where again $u_{\varphi}=\bar{N} \cos \varphi, v_{\varphi}=\bar{N} \sin \varphi$. The behavior of this expression for $\bar{N}=10$ is shown in Fig. [5] (dashed line), where it is compared with the corresponding value of $\left\langle\hat{J}_{z}\right\rangle^{\text {inv }}$ obtained from the numerical solution of the dynamics driven by the exact Hamiltonian of Eq. (1) and in the presence of cavity damping (full line) for the same parameters chosen in Fig. 4(a).

The signal is again periodic with period equal to $\pi$, but it now shows clear peaks at $\varphi=0, \pm \pi$, where it reaches the maximum value $\bar{N} / 2$. The width of the peaks is again inversely proportional to $\bar{N}$, because Eq. (26) can be approximated at small $\varphi$ as

$$
\left\langle\hat{J}_{z}\right\rangle^{\text {inv }} \simeq \frac{\bar{N}}{2} \exp \left(-\frac{\bar{N} \varphi^{2}}{2}\right) \cos ^{2}\left[\frac{\varphi(\bar{N}+1)}{2}\right]
$$

The explicit expression of the phase sensitivity of Eq. (16) can be obtained from Eq. (26) and from Eq. (23) for $\left\langle\hat{J}_{z}^{2}\right\rangle$, which is valid also in the presence of the inversion. The resulting expression is again a cumbersome function of $\varphi$, now achieving its minimum at $|\varphi| \simeq \pi /(4 \bar{N}+4)$, where $\Delta \varphi \simeq 1.45 / \bar{N}$. Differently from the preceding scheme without inversion, the point of minimum phase uncertainty does not coincide with the point of maximum slope of the signal $\left\langle\hat{J}_{z}\right\rangle^{\text {inv }}$, which is again approximately equal to $|\varphi| \simeq \pi /(2 \bar{N}+2)$, as in the scheme with no inversion of time evolution of the preceding Section. At these values of $\varphi$, the phase uncertainty is larger, but still scales as the Heisenberg limit with the number of atoms, because it is $\Delta \varphi \simeq 1.65 / \bar{N}$.

The inversion of the time evolution in $C_{2}$ becomes of crucial importance in the non-dispersive regime. 
In this case, one also has to change the sign of $\hat{J}_{ \pm}$in addition to reversing the sign of $\delta_{2}$ in order to get $\hat{H}_{I, 2}(t) \rightarrow-\hat{H}_{I, 2}(t)$. This second inversion can be realized by applying two opposite $\pi$ Stark shifts. A first one, described by the operator $\exp \left\{i \pi \hat{J}_{z}\right\}$, must be applied in $R_{2}$ just before the entrance in $C_{2}$, while the second, described by the operator $\exp \left\{-i \pi \hat{J}_{z}\right\}$, must be applied in $R_{3}$ soon after the exit from $C_{2}$. The new unitary operators for the Ramsey zones $R_{2}$ and $R_{3}$ then read:

$$
\begin{gathered}
U_{R_{2}}^{\prime}(\varphi)=e^{i \pi \hat{J}_{z}} U_{R_{2}}(\varphi), \\
U_{R_{3}}^{\prime}=U_{R_{3}} e^{-i \pi \hat{J}_{z}} .
\end{gathered}
$$

As a result of the inversion, the narrow central interference peak at $\varphi=0$ becomes much more robust and it survives even outside the dispersive regime of slow velocities. This behavior of $\left\langle\hat{J}_{z}\right\rangle^{\text {inv }}$ is shown in Fig. 6, for different values of $\bar{N}$, while in Fig. 6)(b) the dependency $\Delta \varphi \simeq 2.0 / \bar{N}$ is clearly visible. This fact proves that thanks to inversion in $C_{2}$, we are able to reach about one half of the ultimate, Heisenberg-limited, resolution for the estimate of $\varphi$, even in the non-dispersive regime of fast atoms.

Notice that in the non-dispersive regime the dynamics is no more described by the Hamiltonian of Eq. (2) and therefore the atomic phase shift is no more given by Eq. (6). Nonetheless, we have still used Eq. (6) for the choice of the value of $\delta$ at a given velocity $v$, because it turned out in the numerical simulation to give the best results. When fast atoms are used $(v=180 \mathrm{~m} / \mathrm{s})$, the atomic state after the interactions in $C_{1}$ and $C_{2}$ is rather different from that of the dispersive limit [Eqs. (8) and (12)]. However, the interference experiment in the presence of the inversion in $C_{2}$ still approaches the Heisenberg limit.

The main experimental limitations affecting this scheme are cavity damping, the spontaneous emission of the atoms and the non-unit detection efficiency. Cavity damping is not a serious limitation because the cavities play a passive role in the experiment, only allowing the virtual exchange of excitations between the atoms. The cavities are initially in the vacuum state and can get photons from the excited Rydberg atoms only close to resonance. Cavity decay starts to significantly affect the generated maximally entangled GHZ atomic state only when $\gamma_{\mathrm{c}} \simeq \delta \simeq \Omega_{0} \sqrt{N}$. This is not a strong condition on $\gamma_{\mathrm{c}}$ because typically $\delta \simeq 10^{6}$ $\mathrm{s}^{-1}$. Thus, a moderate quality factor of $Q=\omega_{e g} / \gamma_{\mathrm{c}}>10^{6}$ would be sufficient, compared to experimentally attainable values of $Q>10^{8}$.

Also atomic spontaneous emission does not represent a serious problem, thanks to the use of circular Rydberg levels with lifetimes of the order of $30 \mathrm{~ms}$, even though it puts a practical upper bound on the maximum number of atoms $N$. Spontaneous emission implies a decoherence timescale of the maximally entangled atomic GHZ states of the order of 30/N ms. This decoherence time has to be longer than the time of flight through the apparatus which is of the order of $1 \mathrm{~ms}$. Therefore it is reasonable to perform the experiment with up to 20 atoms.

To consider a non-ideal detection efficiency, we assume that the detector is characterized by the same quantum efficiency $\eta$ for detecting atoms in $e$ or $g$, that there are no dark counts, and we neglect the possibility of a wrong state detection $(e \leftrightarrow g)$, the phase uncertainty simply reads $\Delta \varphi^{\prime}=\eta^{-1} \Delta \varphi$. Typical detection efficiencies attainable in a cavity QED experiment with Rydberg atoms are between $80 \%$ and $100 \%$ 23].

\section{Conditioning to the number of detected atoms}

In the preceding schemes, we did not use any information on the number of detected atoms. We now show that we can improve the phase sensitivity by means of an appropriate data processing of the signals conditioned to the number of detected atoms. If one post-selects only the data corresponding to a given number of detected atoms $N_{d}=N_{e}^{\text {det }}+N_{g}^{\text {det }}$, in the ideal case of a detector with quantum efficiency $\eta=1$, one would get an interference signal coinciding with the ideal one [Eq. (15)] with $N=N_{d}$ (even), thanks to the conditional generation of a maximally entangled GHZ state of $N_{d}$ atoms. If instead the detector is not perfect, the interference signal is the result of an average over many experimental runs in which the actual number of atoms is equal or larger than $N_{d}$. As a consequence, the contrast of the interference fringes 
rapidly worsens for a decreasing detection efficiency $\eta$. This post-selection strategy is in a certain sense opposite to the one considered in the preceding sections, where we have averaged the interferometric signal over all the runs, regardless the value of the number of detected atoms. In the post-selection case, one gives zero weight to the signal conditioned to a number of detected atoms $N_{d}$ different from the selected one. The Poissonian-averaged signal of the preceding section instead corresponds to keep all the data and to give the same weight to the conditioned signals.

It is evident that the post-selection strategy is not optimal for achieving the maximum phase sensitivity, because it implies wasting most of the resources, i.e., all the atoms of the runs with a number of detected atoms different from the selected one $N_{d}$. In this experiment, the best strategy for achieving the maximum phase sensitivity is to keep all the data, however grouping them into different sets according to the corresponding number of detected atoms $N_{d}$, and then give appropriate weights $w\left(N_{d}\right)$ to the signal conditioned to the detection of $N_{d}$ atoms, $\left\langle\hat{J}_{z}\left(N_{d}\right)\right\rangle^{\text {cond }}$. This corresponds to consider the following interferometric signal

$$
\left\langle\hat{J}_{z}\right\rangle^{w}=\sum_{N_{d}=0}^{\infty} P\left(N_{d}\right) w\left(N_{d}\right)\left\langle\hat{J}_{z}\left(N_{d}\right)\right\rangle^{c o n d}
$$

where $P\left(N_{d}\right)$ is the probability of detecting $N_{d}$ atoms which, due to the assumptions made above, is given by

$$
P\left(N_{d}\right)=e^{-\eta \bar{N}} \frac{(\eta \bar{N})^{N_{d}}}{N_{d} !} .
$$

The optimized signal corresponds to take the weights $w^{\text {opt }}\left(N_{d}\right)$ which, for each $\varphi$, minimize the phase uncertainty $\Delta \varphi$ of Eq. (16), which for the signal of Eq. (30) has the explicit form

$$
\Delta \varphi=\frac{\left[\sum_{N_{d}=0}^{\infty} P\left(N_{d}\right) w\left(N_{d}\right)^{2}\left\langle\hat{J}_{z}^{2}\left(N_{d}\right)\right\rangle^{\text {cond }}-\left(\sum_{N_{d}=0}^{\infty} P\left(N_{d}\right) w\left(N_{d}\right)\left\langle\hat{J}_{z}\left(N_{d}\right)\right\rangle^{c o n d}\right)^{2}\right]^{1 / 2}}{\left|\sum_{N_{d}=0}^{\infty} P\left(N_{d}\right) w\left(N_{d}\right) \partial\left\langle\hat{J}_{z}\left(N_{d}\right)\right\rangle^{c o n d} / \partial \varphi\right|} .
$$

The Poisson-averaged signal of the preceding sections corresponds to the particular case $w\left(N_{d}\right)=1 \forall N_{d}$ in Eq. (30).

We illustrate this optimization strategy by applying it to the experiment with the inversion of time evolution in $C_{2}$, in the dispersive limit of large detuning. In this limit, using Eqs. (15) and (25), it is possible to find, after long but straightforward calculations, that the conditional signal is given by

$$
\begin{aligned}
\left\langle\hat{J}_{z}\left(N_{d}\right)\right\rangle^{\text {cond }}=\frac{N_{d}}{4} e^{-\bar{N}_{l}}\{ & e^{\bar{N}_{l} \cos \varphi}\left[\cos \left(N_{d} \varphi+\bar{N}_{l} \sin \varphi\right)+(\cos \varphi)^{N_{d}-1}\right] \\
& \left.+(-1)^{N_{d}} e^{-\bar{N}_{l} \cos \varphi}\left[\cos \left(N_{d} \varphi-\bar{N}_{l} \sin \varphi\right)-(\cos \varphi)^{N_{d}-1}\right]\right\},
\end{aligned}
$$

where $\bar{N}_{l}=\bar{N}(1-\eta)$, is the mean number of atoms lost by the detector. Moreover in the dispersive limit one also finds

$$
\left\langle\hat{J}_{z}^{2}\left(N_{d}\right)\right\rangle^{\text {cond }}=\frac{N_{d}^{2}}{4}+\frac{N_{d}-N_{d}^{2}}{8} \sin ^{2} \varphi\left[1-(-1)^{N_{d}} e^{-\bar{N}_{l}}\right]
$$

These two latter expressions are then inserted into the expression for the phase uncertainty $\Delta \varphi$ of Eq. (32), which is then minimized with respect to the weights $w\left(N_{d}\right)$. The corresponding optimal weights $w^{\text {opt }}\left(N_{d}\right)$ depend upon the phase shift applied in $R_{2}, \varphi$, due to the dependence of $\Delta \varphi$ upon $\varphi$, and when inserted 
(a)

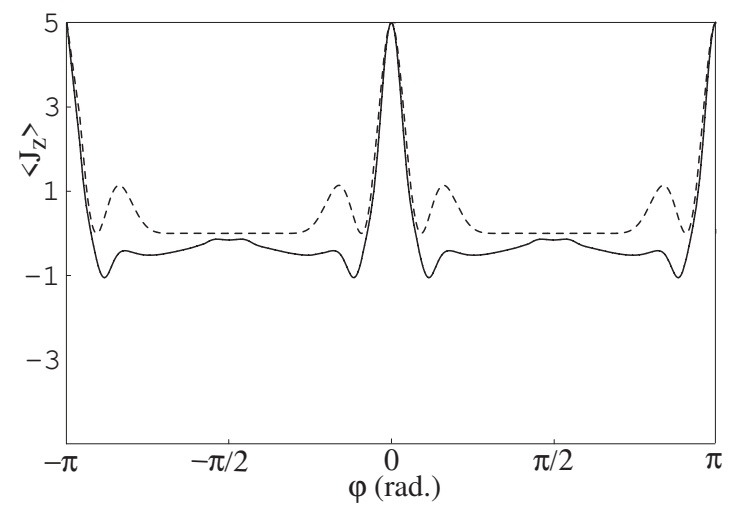

(b)

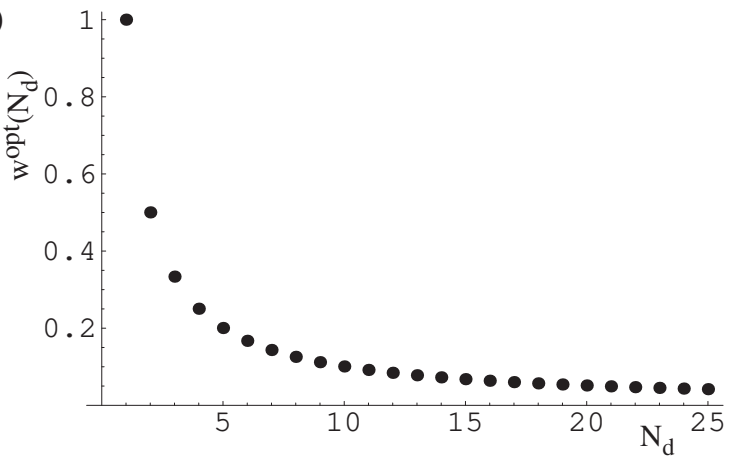

Figure 7. (a) Optimal interferometric signal $\left\langle\hat{J}_{z}\right\rangle^{\text {opt }}$ of Eq. 35] (full line) versus the phase shift applied in $R_{2}, \varphi$. The optimal weights are determined by the condition of minimum phase uncertainty at each $\varphi$. The dashed line refers to the Poisson-averaged signal of Eq. [26], corresponding to take equal weights. (b) Weights $w^{\mathrm{opt}}\left(N_{d}\right)$ of the optimal signal versus $N_{d}$ at the point of the maximum phase sensitivity. The plots refer to $\eta=0.8$ and $\bar{N}=12.5$ and to the dispersive limit of large detuning, where Eqs. 33. 34 and apply.

into Eq. (30), one gets the optimal interference signal

$$
\left\langle\hat{J}_{z}\right\rangle^{\mathrm{opt}}=\sum_{N_{d}=0}^{\infty} P\left(N_{d}\right) w^{\mathrm{opt}}\left(N_{d}\right)\left\langle\hat{J}_{z}\left(N_{d}\right)\right\rangle^{\mathrm{cond}}
$$

This optimized signal is shown in Fig. Z(a) (full line), which refers to the case $\eta=0.8$ and $\bar{N}=12.5$. The corresponding minimum phase uncertainty is a function of $\varphi$, and its minimum value $\Delta \varphi_{\text {min }}$ is closer to the ideal Heisenberg limit: $\Delta \varphi_{\min } \simeq 1.3 / \eta \bar{N}$. Comparing with the result of the preceding section, we see that optimizing the information provided by conditioning on the number of detected atoms yields an improved phase sensitivity, since the corresponding minimum phase uncertainty of the Poisson-averaged case scales as $\Delta \varphi_{\min } \simeq 1.45 / \eta \bar{N}$. As it happens in the Poisson-averaged signal, the best phase sensitivity is achieved at a phase shift $\varphi$ smaller than that corresponding to the maximum slope of the optimal signal $\left\langle\hat{J}_{z}\right\rangle^{\text {opt }}$, which is approximately equal to $|\varphi| \simeq \pi /(3 \eta \bar{N})$. At this value of the phase shift, the phase uncertainty still scales as the Heisenberg limit, but is larger and it is roughly given by $\Delta \varphi \simeq 1.5 / \eta \bar{N}$.

It is also interesting to see the behavior of the optimal weights $w^{\text {opt }}\left(N_{d}\right)$ at the phase shift $\varphi$ corresponding to the maximum phase sensitivity, which are shown in Fig. Z(b), again for the case $\eta=0.8$ and $\bar{N}=12.5$. One can notice that they monotonically increase for decreasing $N_{d}$ and this is due to the fact that the optimal weights tends to be inversely proportional to $\left\langle\hat{J}_{z}^{2}\left(N_{d}\right)\right\rangle^{\text {cond }}$. From the analysis of Section II, one could have expected an oscillating behavior of $w^{\mathrm{opt}}\left(N_{d}\right)$, with maxima corresponding to $N_{d}$ even and minima corresponding to $N_{d}$ odd. However it can be seen that this happens only in the limit $\eta \rightarrow 1$, when the probability to detect a wrong parity of the number of atoms becomes negligible. Only in this limit, one can safely discriminate between an even and an odd number of atoms and suppress the contribution of the runs with an odd number of atoms.

\section{Conclusions}

We have presented a Ramsey-like interference experiment for a cavity-QED system, able to reach the ultimate Heisenberg limit for the estimation of an atomic phase-shift. We considered a system of $N$ twolevel Rydberg atoms successively crossing two microwave cavities. A dispersive atom cavity interaction is able to generate an atomic Schrödinger cat, i.e., a superposition of two atomic coherent states, which represents a maximally entangled state of the $N$ atoms. Using this state, we designed an interference experiment yielding fringes $N$ times narrower than those one would have obtained if $N$ disentangled atoms were used.

As discussed in Sec. 3, the most important limitations affecting the the experiment are the fluctuations of the number of Rydberg atoms, and the non-unit detection efficiency. Despite these limitations, 
one can approach Heisenberg-limited sensitivity, because a narrow central fringe, with a width inversely proportional to $\bar{N}$, survives even when averaged over the fluctuations of the number of detected atoms.

Finally we have considered a conditional scheme in which we post-select only the events with a fixed number of detected atoms. In such a case, the best strategy for achieving the maximum phase sensitivity is to keep all the data, however grouping them into different sets according to the corresponding number of detected atoms $N_{d}$, and then give appropriate weights $w\left(N_{d}\right)$ to the signal conditioned to the detection of $N_{d}$ atoms, $\left\langle\hat{J}_{z}\left(N_{d}\right)\right\rangle^{\text {cond }}$. One can then determine the optimal weights maximizing the phase sensitivity and the corresponding optimized signal closely approaches the ultimate Heisenberg limit, because the minimum phase uncertainty is $\Delta \varphi_{\min } \simeq 1.3 / \eta \bar{N}$.

The proposed experiment should allow for the first time to achieve the Heisenberg limit for spectroscopy with a larger number of atoms compared to the experiments realized so far with entangled photons or ions. Despite this application in spectroscopy, characterizing the maximally entangled Schrödinger cat state by e.g. state tomography and monitoring its decoherence would be a very interesting perspective.

\section{Acknowledgements}

We acknowledge the funding by the city of Paris (D.V.), by a Marie-Curie fellowship of the European Community (S.K.) and by the EU under the IP projects "QGATES" and "SCALA".

\section{References}

[1] W.M. Itano et al., Phys. Rev. A 47, 3554 (1993); D.J. Wineland, J.J. Bollinger, W.M. Itano, F.L. Moore and D.J. Heinzen, Phys. Rev. A 46, R6797 (1992); G.S. Agarwal and R.R. Puri, Phys. Rev. A 49, 4968 (1994).

[2] D.J. Wineland, J.J. Bollinger, W.M. Itano and D.J. Heinzen, Phys. Rev. A 50, 67 (1994).

[3] C.M. Caves, Phys. Rev. D 23, 1693 (1981); B. Yurke, Phys. Rev. Lett. 56, 1515 (1986); M. Xiao, L.-A. Wu and H.J. Kimble, ibid. 59, 278 (1987); M.J. Holland and K. Burnett, ibid. 71, 1355 (1993); B. C. Sanders and G. J. Milburn, ibid. 75, 2944 (1995); Z.Y. Ou, Phys. Rev. A 552598 (1997); A. Luis and L.L. Sanchez-Soto, Progress in Optics 41 (Amsterdam: Elsevier) p. 421, (2000); A. Luis, Phys. Rev. A 65025802 (2002).

[4] B. Yurke, S.L. McCall and J.R. Klauder, Phys. Rev. A 33, 4033 (1986).

[5] M. Kitagawa and M. Ueda, Phys. Rev. A 47, 5138 (1993).

[6] C.G. Gerry, A. Benmoussa, A.R. Campos, Phys. Rev. A 66, 013804 (2002).

[7] J.J. Bollinger, W.M. Itano, D.J. Wineland, and D.J. Heinzen, Phys. Rev. A 54, R4649 (1996).

[8] D. Leibfried et al., Science 304, 1476 (2004).

[9] D. Leibfried et al., Nature (London) 438, 639 (2005).

[10] V. Giovannetti, S. Lloyd, and L. Maccone, e-print quant-ph/0509179

[11] M.W. Mitchell, J.S. Lundeen, and A.M. Steinberg, Nature (London) 429, 161 (2004).

[12] P. Walther, J-W. Pan, M. Aspelmeyer, R. Ursin, S. Gasparoni, and A. Zeilinger, Nature (London) 429, 158 (2004).

[13] N.F. Ramsey, Molecular Beams (Oxford, London, 1963), p. 124.

[14] C. Genes, R.P. Berman, A.G. Rojo, Phys. Rev. A 68, 043809 (2003).

[15] G.S. Agarwal, R.R. Puri, R.P. Singh, Phys. Rev. A 56, 2249 (1997).

[16] J.M. Raimond, M. Brune, and S. Haroche, Rev. Mod. Phys. 73, 565 (2001).

[17] R.H. Dicke, Phys. Rev. 93, 99 (1954).

[18] M. Tavis, F.W. Cummings, Phys. Rev. 170, 379 (1968).

[19] K. Mølmer and A.S. Sørensen, Phys. Rev. Lett. 82, 1835 (1999).

[20] B. Yurke and D. Stoler, Phys. Rev. Lett. 57, 13 (1986).

[21] F.T. Arecchi, E. Courtens, R. Gilmore, and H. Thomas, Phys. Rev. A 6, 2211 (1972).

[22] D. Leibfried et al., Phys. Rev. Lett 89, 247901 (2002).

[23] P. Maioli et al., Phys. Rev. Lett. 94, 113601 (2005). 